\title{
Graphical User Interface Calculator for FBGS Performance Optimization Based on New Window Formulas
}

\author{
Calculadora Gráfica de Interfaz de Usuario para Optimizar \\ el Rendimiento de FBGS Basado en Nuevas Fórmulas de Ventana
}

\author{
F. Chaoui ${ }^{1}$, O. Aghzout ${ }^{2}$, M. El Yakhloufi ${ }^{3}$ \\ 1. Dept. of Physics, Faculty of Sciences, UAE, Tetouan, Morocco \\ 2. Electronics \& Microwaves Group, Telecommunication Dept., ENSA, UAE, Tetouan, Morocco \\ 3. Condensed Matter Physics Group, Faculty of Sciences, UAE, Tetouan, Morocco \\ ${ }^{(*)}$ E-mail: fahdchaoui@outlook.com
}

Received: 28/07/2017_Accepted: 07/05/2018

DOI: 10.7149/OPA.51.2.50005

\begin{abstract}
:
In this research paper, we develop on one hand a novel theoretical approach to optimize the performance of Fiber Bragg Grating Sensor (FBGS) based on an automated MATLAB-based program, and on the other hand, we propose a new graphical user interface including all design parameters of the FBGS to optimize automatically its window profile based on the sensing system requirement. The main objective of this approach is to obtain the higher reflected power from the FBGS as long as side lobes are kept below the required level. All formulas of the proposed method of optimization are programmed using the Coupled Matrix Theory (CMT) and the Transfer Matrix Method (TMM), which describe the light behavior within the FBGS. The proposed easy-to-use graphical interface allows also researchers to determine easily all parameters required for high FBGS performance providing a high precision and accuracy of numerical results. The proposed theoretical approach has been compared with the window profiles proposed by other authors. All results demonstrate the efficiency of the proposed method.
\end{abstract}

Key words: Fiber Bragg Grating Sensor (FBGS), Coupled Mode Theory (CMT), Side Lobe Level (SLL), Transfer Matrix Method (TMM).

\section{REFERENCES AND LINKS / REFERENCIAS Y ENLACES}

[1] L. Alwis, T. Sun, K.T.V. Grattan, "Developments in optical fiber sensors for industrial applications," Optics \& Laser Technology, 78, 62-66 (2016).

[2] E. Al-Fakih, N. A. Abu Osman, F. R. M. Adikan, "The Use of Fiber Bragg Grating Sensors in Biomechanics and Rehabilitation Applications: The State-of-the-Art and Ongoing Research Topics," Sensors, 12 12890-12926 (2012).

[3] S. Udoh, J. Njuguma, R. Prabhu, "Modelling and Simulation of Fiber Bragg Grating Characterization for Oil and Gas Sensing Applications", First International Conference on Systems Informatics, Modelling and Simulation (2014).

[4] A. Minardo, A. Cusano, R. Bernini, "Response of Fiber Bragg Gratings to Longitudinal Ultrasonic Waves,” IEEE transactions on ultrasonics, ferroelectrics, and frequency control, 52, no. 2, (2005).

[5] E. Gemzický, J. Müllerová, "Analysis of simulated reflection characteristics of uniform and apodized fiber Bragg gratings," Advances in Electrical and Electronic Engineering (2008).

[6] N. A. Mohammed, T. A. Ali, M. H. Aly, "Evaluation and performance enhancement for accurate FBG temperature sensor measurement with different apodization profiles in single and quasi-distributed DWDM systems," Optics and Lasers in Engineering, 55, pp. 22-34 (2014). 
[7] Y. Yang, G. Wang, "Analyze and model to chirped fiber grating with new apodization function," Proceedings of the Third International Symposium on Computer Science and Computational Technology, Jiaozuo, P. R. China, 14-15, pp. 312-314, (2010).

[8] D. T. H. Tan, K. Ikeda, R. E. Saperstein, B. Slutsky, Y. Fainman, "Chip-scale dispersion engineering using chirped vertical gratings," Optics Letters 33, No. 24, (2008).

[9] I. Yulianti, A. S. M. Supa'at, S. M. Idrus, A. M. Al-hetar, "Simulation of Apodization Profiles Performances for unchirped Fiber Bragg Gratings," IEEE 978-1-4244-7187-4, (2010).

[10] H. Venghaus, Wavelength filters in fibre optics, Springer, Chapter 5, 3-540-31769-4 (2006).

\section{Introduction}

Optical sensors are the forefront of research in optics nowadays. Various approaches and technologies have been utilized for measuring a number of different physical parameters based on optical sensors such as strain, temperature and pressure $[1,2]$. In fact, the future of optical sensors is very promising because they exhibit very competitive advantages such as immunity to electromagnetic interference and to ionizing radiation ( $\gamma$-ray, X-ray etc.). These characteristics make optical fiber sensors key sensing devices in radiative environments, like high-speed trains, aircraft and nuclear power plants, where the detection and evaluation of pressure and temperature changes are very crucial [3]. In particular, Fiber Bragg Grating (FBG) has attracted noticeable interest as one of the most important recent developments in optical sensors technology. FBGs are now commercially available and they find extensive application in many areas such as sensing applications, filtering and dispersion compensation of optical signals. The important feature of FBG sensors is that the sensed physical change is transmitted as a wavelength shift, giving reproducible measurements in spite of optical losses and intensity fluctuations [4]. Per contra, FBGs with uniform window profile show reflection spectrum with high side lobes, besides highly nonlinear dispersion characteristics, which makes them unsuitable for high performance applications [5]. Therefore, different window profiles have been recently developed and applied for FBGS technology as they provide superior filtering performance and low side lobe levels [6,7]. Nevertheless, strong windowing process results in low reflectivity level and wastes an important amount of reflectivity power [8]. That is why; the choice of the FBGS window profile has to be smartly designed according to the sensing system requirement. In this work, we propose a graphical user interface calculator for a novel theoretical approach to optimize the window profile of FBGS based on an automated MATLAB-based new code. The main goal of the proposed program is to seek the higher reflectivity possible as long as side lobes are kept at the required level. All formulas of the proposed method of optimization are programmed using the Coupled Matrix Theory (CMT) and the Transfer Matrix Method (TMM), which describe the light behavior within the FBGS. The proposed algorithm optimize automatically the FBGS window profile according to the SLL of the FBGS reflection response. The rest of the paper is organized as follows: In Section II, we briefly describe the general theoretical model used for the calculation of the grating response. The main window profiles considered in this paper are also presented in this section. In Section III, we illustrate our proposed Graphical User Interface and the developed calculation tools that it offers, then we compare simulation results of our proposed method with the window profiles proposed by other authors to prove how the proposed method helps to obtain better results. The performance of the proposed models is further optimized to seek $100 \%$ reflectivity, which cannot be achieved using the state-of-art alternatives. The conclusions are given in Section IV.

\section{Theory and modeling}

\section{2.a. Apodization and Reflectivity:}

The FBG is completely characterized by its refractive index distribution along the fiber [9]:

$$
n_{e f f}(z)=n_{0}+f(z) \cdot \Delta n_{a c} \cdot v \cdot \cos \left(\left(\frac{2 \pi}{\Lambda}\right) z+\theta(z)\right)
$$

where $z$ is the position, $n_{0}$ is the refractive index prior to grating inscription, $\Lambda$ is the grating period, $\Delta n_{a c}$ is the refractive index modulation amplitude, $f(z)$ is the apodization function, $\theta(z)=\frac{2 \pi}{\Lambda} C z^{2}$ is the chirp function where $C$ is the chirp parameter, and $v$ is the fringe visibility. The FBG reflect the propagated light in fiber according to Bragg wavelength, which is given as follow:

$$
\lambda_{B}=2 n_{\text {eff }} \Lambda
$$


where $n_{\text {eff }}$ is the effective refractive index of the core of the fiber and $\Lambda$ is the grating period. In this work, the well-known Transfer Matrix Method (TMM) is applied to solve the Coupled Mode Equations (CME) that describes the behavior of light propagation in FBG where the two backward and forward propagating waves inside the grating structure are related by coupled differential equations. Fig. 1 illustrates the electrical field distribution within the FBG.

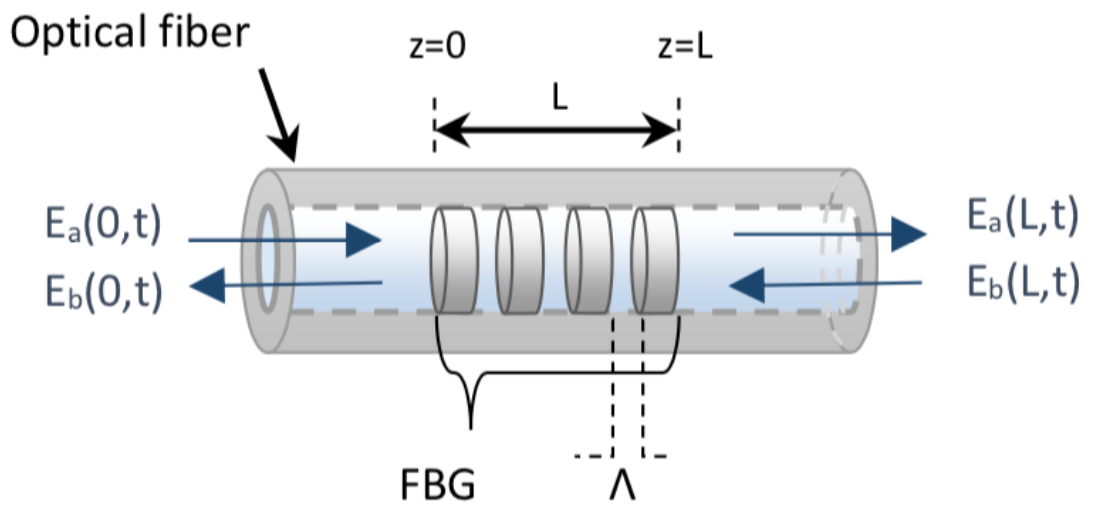

Fig.1. The forward and backward propagating waves within the FBG

For sinusoidal variations of the index perturbation, the coupling coefficient $\mathrm{k}$ is given by:

$$
K(z)=\frac{\pi}{\lambda} \Delta n_{a c} \cdot f(z) \cdot v
$$

To solve the couple mode equations and to obtain the spectral response of FBGS the well-known Transfer Matrix Method (TMM) should be taken into account. In this approach, the grating is divided into $\mathrm{N}$ uniform sections; each section is represented by a $2 \times 2$ matrix. By multiplying these matrices, a global matrix that describes the whole grating is obtained. By applying the appropriate boundary conditions and by solving the coupled-mode equations, we find the following transfer matrix relation between the fields at $\mathrm{z}$ and at $z+\Delta$.

$$
\left[\begin{array}{l}
A(z) \\
B(z)
\end{array}\right]=\left[\begin{array}{cc}
\cosh (\gamma \Delta)-i \frac{\Delta \beta}{\gamma} \sinh (\gamma \Delta) & -i \frac{\mathrm{k}}{\gamma} \sinh (\gamma \Delta) \\
i \frac{\mathrm{k}}{\gamma} \sinh (\gamma \Delta) & \cosh (\gamma \Delta)+i \frac{\Delta \beta}{\gamma} \sinh (\gamma \Delta)
\end{array}\right]\left[\begin{array}{l}
A(z+\Delta) \\
B(z+\Delta)
\end{array}\right]
$$

where $\gamma=\sqrt{\mathrm{k}^{2}-\Delta \beta^{2}}$ is phase shift to Bragg wavelength $\lambda_{B}$ and $\Delta=L / N$ is section length. We can connect the fields at the two ends of the grating through:

$$
\left[\begin{array}{l}
A(0) \\
B(0)
\end{array}\right]=T\left[\begin{array}{l}
A(L) \\
B(L)
\end{array}\right]
$$

where

$$
T=T_{1} * T_{2} * \ldots T_{j} \ldots T_{N-1} * T_{N}
$$

The matrix $T_{j}$ is the transfer matrix for one section written in (a):

$$
T_{j}=\left[\begin{array}{cc}
\cosh \left(\gamma l_{j}\right)-i \frac{\Delta \beta}{\gamma} \sinh \left(\gamma l_{j}\right) & -i \frac{\mathrm{k}}{\gamma} \sinh \left(\gamma l_{j}\right) \\
i \frac{\mathrm{k}}{\gamma} \sinh \left(\gamma l_{j}\right) & \cosh \left(\gamma l_{j}\right)+i \frac{\Delta \beta}{\gamma} \sinh \left(\gamma l_{j}\right)
\end{array}\right]
$$

As a result, $\mathrm{T}$ is a $2 \times 2$ matrix elements:

$$
T=\left[\begin{array}{ll}
T_{11} & T_{12} \\
T_{21} & T_{22}
\end{array}\right]
$$

Therefore, the FBGS reflectivity is given by: 


$$
R=\left|T_{21} / T_{11}\right|^{2}
$$

More details about CME and TMM are found in [10].

\section{2.b. Window functions}

The most performant window profiles discussed in literature are given as follow [6-8]:

1- Gaussian:

$$
f(z)=\exp \left(-\ln 2 \cdot\left(\frac{z-\frac{L}{2}}{s \cdot \frac{L}{2}}\right)^{2}\right)
$$

2- Blackman:

$$
f(z)=0.42-0.5 \cos (2 \pi z / L)+0.8 \cos (4 \pi z / L)
$$

3- Nuttal:

$$
f(z)=a_{0}-a_{1} \cos \left(2 \pi\left(\frac{z}{L}\right)\right)+a_{2} \cos \left(4 \pi\left(\frac{z}{L}\right)\right)-a_{3} \cos \left(6 \pi\left(\frac{z}{L}\right)\right)
$$

Where $a_{0}=0.3635819, a_{1}=0.4891775, a_{2}=0.1365995, a_{3}=0.0106411$ are the window coefficients, $L$ is the grating length, $z$ is the coordinate of light propagation along the length of FBG.

\section{Results and discussion}

\section{3.a. Apodization and Reflectivity:}

In this work, we propose to use new window profiles that depends on an editable coefficient $\alpha$, which is calculated automatically based on the required SLL of the FBG reflection spectrum, taken into account that SLL should not be higher than $-40 \mathrm{~dB}$ according to ITU standards:

- 2-terms : If the required SLL is higher than - $50 \mathrm{~dB}$

$$
f(z)=\alpha-(1-\alpha) \cos \left(\frac{2 \pi z}{L}\right)
$$

- 3-terms : If the required SLL is higher than -75dB

$$
f(z)=\alpha-0.5 \cos \left(\frac{2 \pi z}{L}\right)+(0.5-\alpha) \cos \left(\frac{4 \pi z}{L}\right)
$$

- 4-terms : If the required SLL is equal to or lower than $-75 \mathrm{~dB}$

$$
f(z)=\alpha-(\alpha+\delta) \cos \left(\frac{2 \pi z}{L}\right)+(0.5-\alpha) \cos \left(\frac{4 \pi z}{L}\right)-(0.5-(\alpha+\delta)) \cos \left(\frac{6 \pi z}{L}\right)
$$

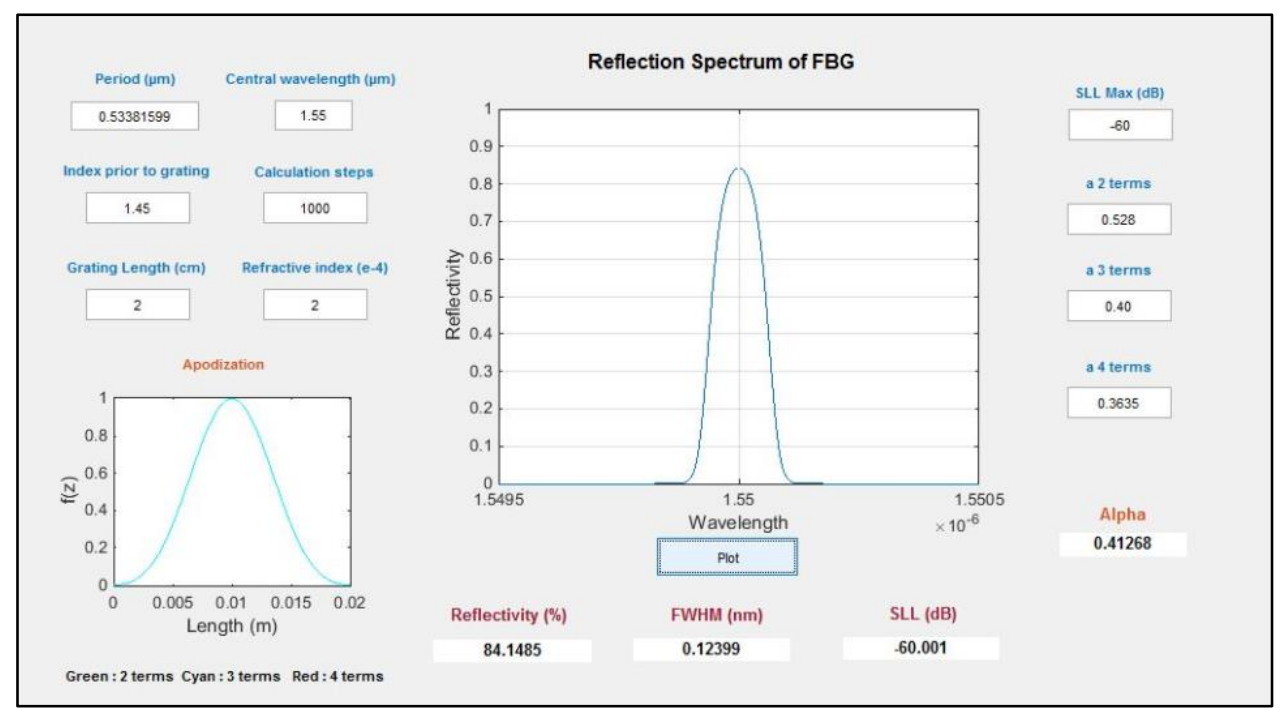

Fig. 2. GUI for the proposed automated MATLAB code for 3-terms profile. 
The developed code presented in this paper allows the user to automatically calculate coefficient $\alpha$ to seek the higher Reflectivity level, as long as SLL and FWHM are kept in acceptable levels according to the sensing system requirement. For good and efficient FBGS, high reflectivity, low side lobes and narrow FWHM should be obtained. The broad spectral response can result in poor sensitivity and hence should be avoided. So when the program plots the Reflection spectrum based on the FBG input properties, SLL and FWHM are calculated to test if it is acceptable to increase $\alpha$. In case SLL is kept below the required SLL and FWHM is kept between $0.1 \mathrm{~nm}$ and $0.2 \mathrm{~nm}$ (according to ITU standards), the program goes to next iteration with the increased $\alpha$. The proposed graphical user interface for the developed code can be shown in Fig. 2 and Fig. 3.

Fig. 2 shows the reflection spectrum of FBG with $S L L_{\max }=-60 \mathrm{~dB}$. The required maximal SLL is higher than $-75 d B$ and lower than $-50 d B$, so the reflection response of the FBGS is calculated with the 3-terms window profile to obtain the higher reflectivity peak. The optimized $\alpha$ for this FBGS design setting is 0.41268 . Fig. 3 shows the reflection spectrum of FBG with $S L L_{\max }=-75 d B$. The required maximal SLL is equal to $-75 d B$, so the reflection response of the FBGS is calculated with the 4-terms window profile to obtain the higher reflectivity peak and respect the required SLL. The optimized $\alpha$ for this FBGS design setting is 0.36913 .

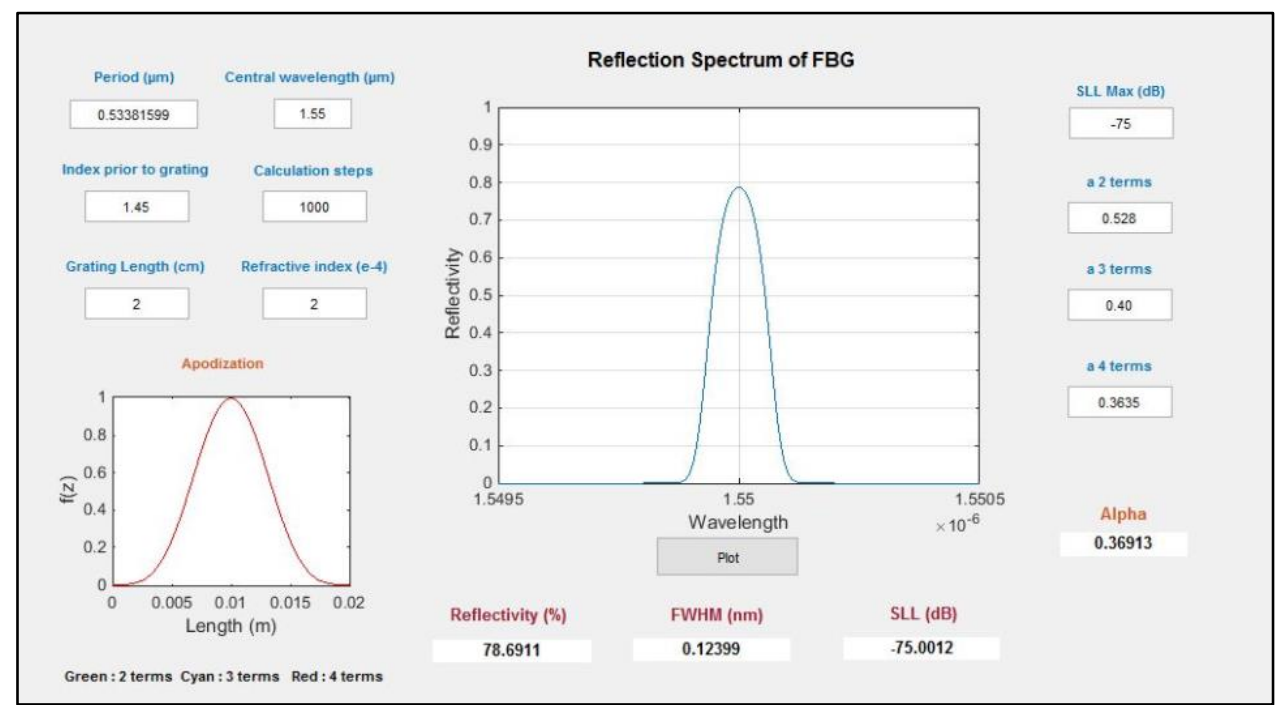

Fig. 3. GUI for the proposed automated MATLAB code for 4-terms profile

Fig. 4 shows the reflection spectrums of the main window profiles discussed in literature (Gaussian, Blackman and Nuttal) compared to Uniform profile. By comparing the 2 spectrums, it can be observed that side lobes are high as expected in Uniform profile, which is not convenient in high performance applications. Nuttal apodization offers better side lobes suppression but waste remarkably the reflected power, which would affects the signal transmission in long distance sensing applications.
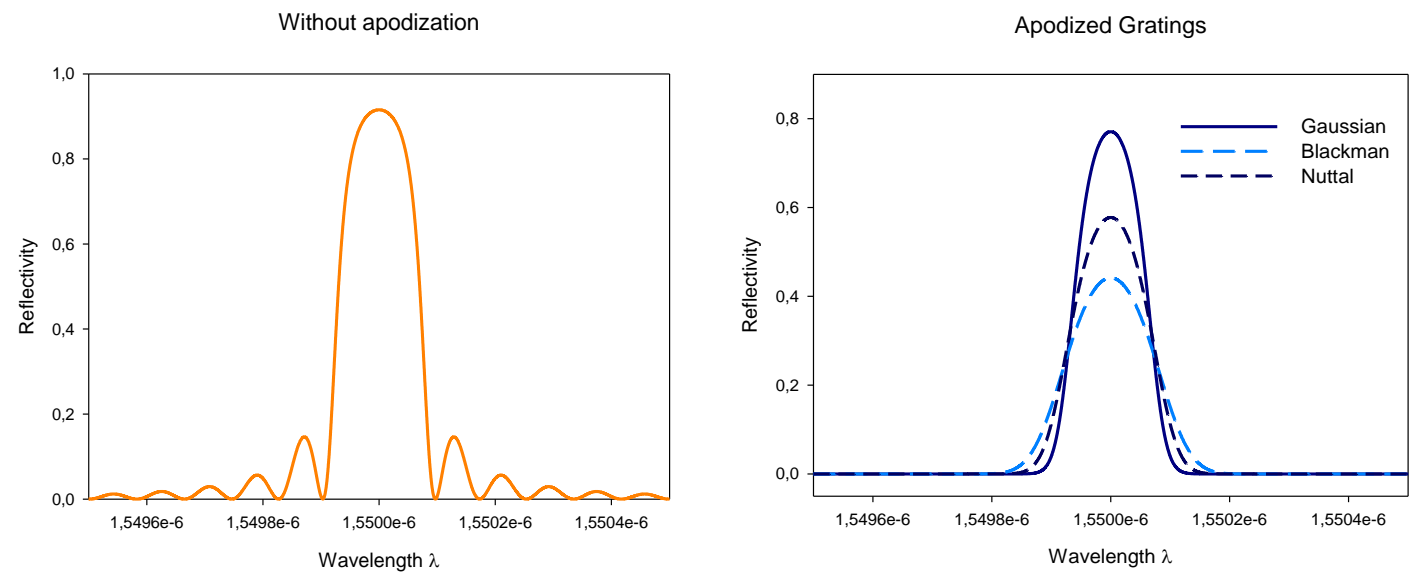

Fig. 4. Reflection spectrums for $\mathrm{L}=1 \mathrm{~cm} d \mathrm{n}=2 \mathrm{e}^{-4}$. 
Fig. 5 illustrates the geometrical shape of the refractive index changes of the proposed window profiles next to the uniform profile based on the grating length of the FBG. It can be seen from Fig. 5 that Uniform profile (without apodization) has the largest geometrical shape compared to the proposed window profiles. It can also be observed that the 2-terms profile has the largest shape between the three proposed window profiles, while 4-terms has the smallest shape, which would affect directly their reflection spectrum.
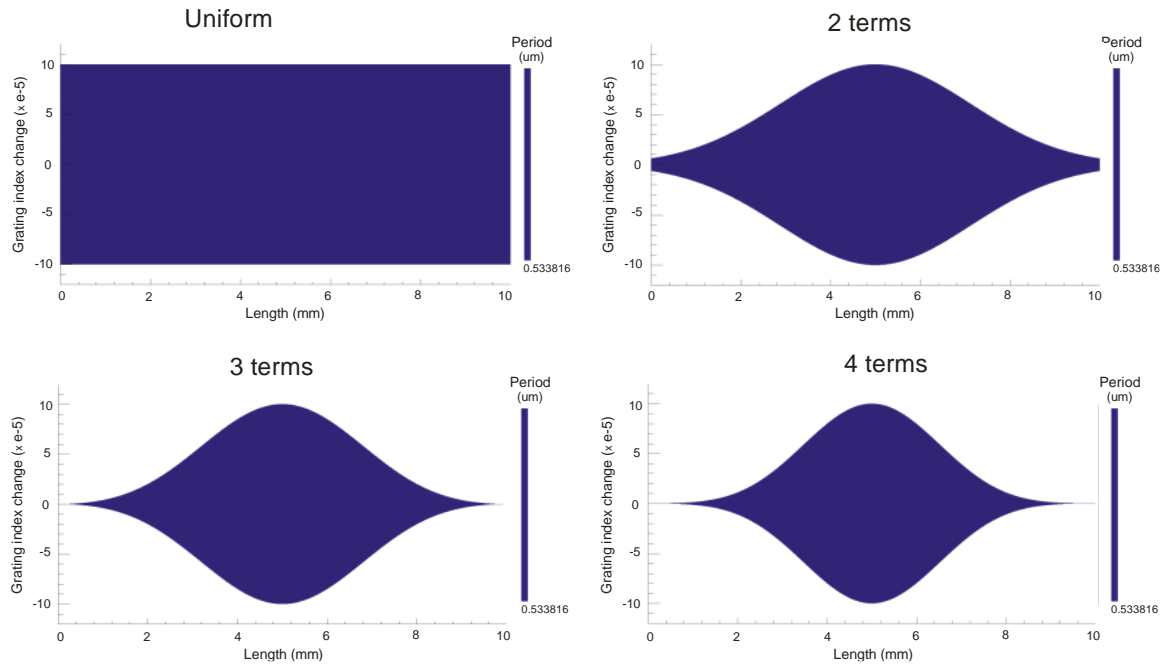

Fig. 5. Geometrical shape of the window profiles

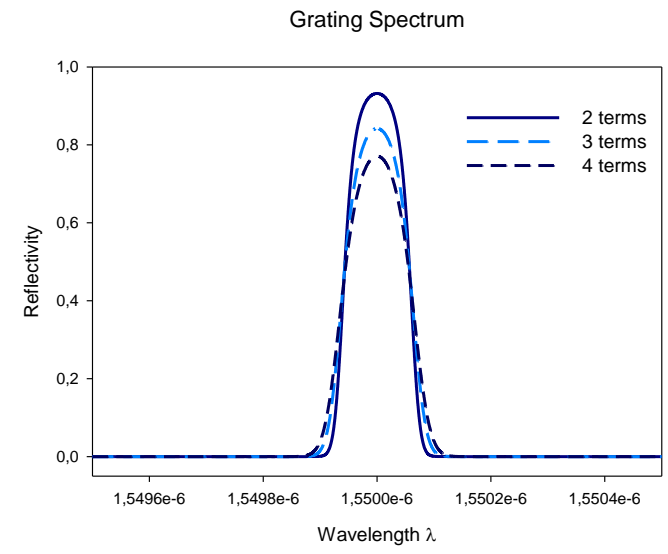

Fig. 6. Reflection spectrums for $\mathrm{L}=2 \mathrm{~cm} d n=2 \mathrm{e}^{-4}$.

Fig. 6 shows the reflection spectrums of 2 -terms, 3 -terms, and 4-terms profiles for $10 \mathrm{~mm}$ grating length and $2 e^{-4}$ refractive index. By comparing the 3 spectrums, it can be observed that 4 -terms has the lowest reflectivity peak due to the strong process of apodization, while the 2 -terms has the highest reflectivity peak. Fig.7.a illustrates the comparison results between the proposed window function with other window profiles proposed by other authors: Gaussian, Blackman and Nuttal [6-8]. The comparison has been carried out using the same initial setting taking into account that the 2-terms, 3-terms and 4-terms profiles respect SLL of $-40 \mathrm{~dB},-50 \mathrm{~dB}$ and $-75 \mathrm{~dB}$ respectively. 
(a)

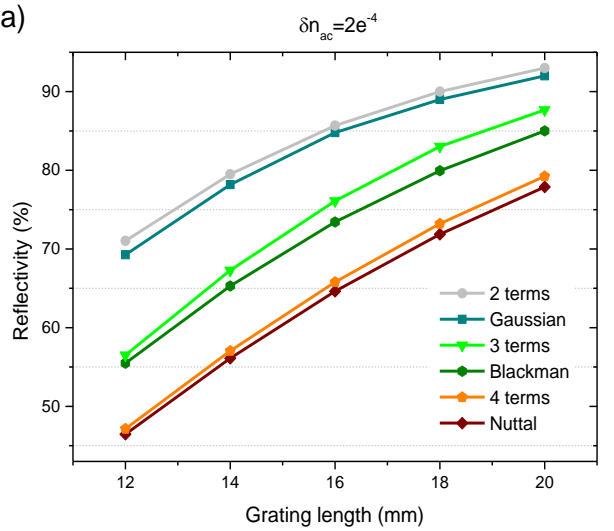

(b)

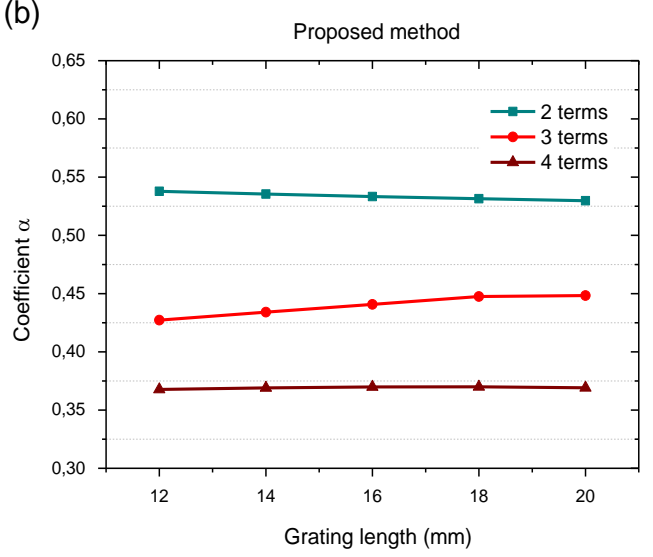

Fig. 7. Comparison results: (a) Reflectivity vs Grating Length (b) Coefficient $\alpha$ vs Grating length.

Fig.7.b shows Coefficient $\alpha$ variations based on the same values of $L$ using the proposed method of apodization. It can clearly be observed from Fig. 7.a that the use of the proposed method provides the higher Reflected power among the different window profiles discussed in literature for all values of grating length, for either 2-terms compared to Gaussian, 3-terms compared to Blackman or 4-terms compared to Nuttal. The proposed window profiles result in obvious superior performance regardless of maximal SLL and FBG length. The variation of coefficient $\alpha$ depends on the FBG design setting as can be shown in Fig. 7.b. When L increases, coefficient $\alpha$ decreases in the 2-terms window profile and increase the 3-terms window profile, whereas it does not follow any regular behavior in the 4-terms window profile, it goes up and down due to the complexed low required SLL.

TABLE 1. Optimized coefficients $\alpha$ for proposed apodization

\begin{tabular}{|c|c|c|c|c|}
\hline$S L L$ & $L(m m)$ & $R(\%)$ & $F W H M(n m)$ & $\alpha$ \\
\hline \multirow{5}{*}{$-40 \mathrm{~dB}$} & 12 & 71.04 & 0.13638 & 0.53784 \\
\hline & 14 & 79.50 & 0.13019 & 0.53551 \\
\hline & 16 & 85.69 & 0.12399 & 0.53329 \\
\hline & 18 & 90.11 & 0.11779 & 0.53144 \\
\hline & 20 & 93.21 & 0.11779 & 0.52970 \\
\hline \multirow{5}{*}{$-50 \mathrm{~dB}$} & 12 & 56.51 & 0.14877 & 0.42718 \\
\hline & 14 & 67.30 & 0.13638 & 0.43405 \\
\hline & 16 & 76.12 & 0.13018 & 0.44069 \\
\hline & 18 & 83.02 & 0.12399 & 0.44750 \\
\hline & 20 & 87.67 & 0.11779 & 0.44832 \\
\hline \multirow{5}{*}{$-75 \mathrm{~dB}$} & 12 & 47.15 & 0.16116 & 0.367719 \\
\hline & 14 & 57.04 & 0.14877 & 0.369000 \\
\hline & 16 & 65.70 & 0.13638 & 0.369904 \\
\hline & 18 & 72.90 & 0.13018 & 0.369923 \\
\hline & 20 & 78.70 & 0.12399 & 0.369132 \\
\hline
\end{tabular}

It can also be noticed from Fig. 7.b that when the required SLL decreases, the optimized coefficient $\boldsymbol{\alpha}$ also decreases from one window profile to another in order to respect the required SLL and seek the higher possible reflectivity peak. Table 1 illustrates the detailed optimized coefficients for the different reflectivity peaks plotted in Fig.7. One can notice in table 1 that all values of FWHM falls in the acceptable range between $0.1 \mathrm{~nm}$ and $0.2 \mathrm{~nm}$, which demonstrates further the reliability of the suggested technique. 


\section{3.b. Performance optimization:}

In this part, the performance of the FBGS is further optimized using the proposed method based on $\boldsymbol{L}$ and $\boldsymbol{d} \boldsymbol{n}$ to find out the most appropriate setting that leads to reflectivity level of $100 \%$, taking into account that none of the state-of-art window profiles leads to $100 \%$ reflectivity while respecting ITU standards (SLL below $-40 \mathrm{~dB}$ and FWHM between $0.1 \mathrm{~nm}$ and $0.2 \mathrm{~nm}$ ). Table 2 shows the best reflectivity levels attained by the three proposed window profiles.

TABLE 2. Performance optimization based grating length

\begin{tabular}{|c|c|c|c|c|c|c|}
\hline \hline Profile & SLL $_{\max }(\mathrm{dB})$ & $\mathrm{L}(\mathrm{cm})$ & $\mathrm{dn}\left(\mathrm{e}^{-4}\right)$ & Optimized $\alpha$ & Reflectivity $(\%)$ & FWHM $(\mathrm{nm})$ \\
\hline \hline 4-terms & -75 & 4.7 & 3.7 & 0.365682 & $99.99 \%$ & 0.1860 \\
\hline 3-terms & -50 & 4.6 & 3.9 & 0.4727 & $100 \%$ & 0.1984 \\
\hline 2-terms & -40 & 4.6 & 3.6 & 0.51081 & $100 \%$ & 0.1798 \\
\hline \hline
\end{tabular}

By comparing the results of the three proposed profiles, reflectivity of $100 \%$ can be achieved while respecting the ITU standards only by using the 3 -terms profile and 2-terms profile, with 0.4727 and 0.51081 as their optimized coefficient $\alpha$ respectively. For the 3 -terms profile, this value is achieved for $L=$ $46 \mathrm{~mm}$ and $d n=3.9 e^{-4}$ with FWHM slightly below $0.2 \mathrm{~nm}$ limit. However, for the 2-terms profile, reflectivity of $100 \%$ is obtained with the same value of grating length, lower refractive index and better FWHM, which makes the 2-terms profile more appropriate for use in complexed sensing applications where high reflected power must be obtained.

Fig. 8 shows the grating spectrum of the optimized FBGS with 100\% reflected power using the 2 -terms profile. It can clearly be seen that there are almost no side lobes (below $-40 \mathrm{~dB}$ ) and very good FWHM (below $0.2 \mathrm{~nm}$ ), which cannot be achieved as mentioned previously using any of the profiles proposed by other authors in literature while respecting the ITU standards.

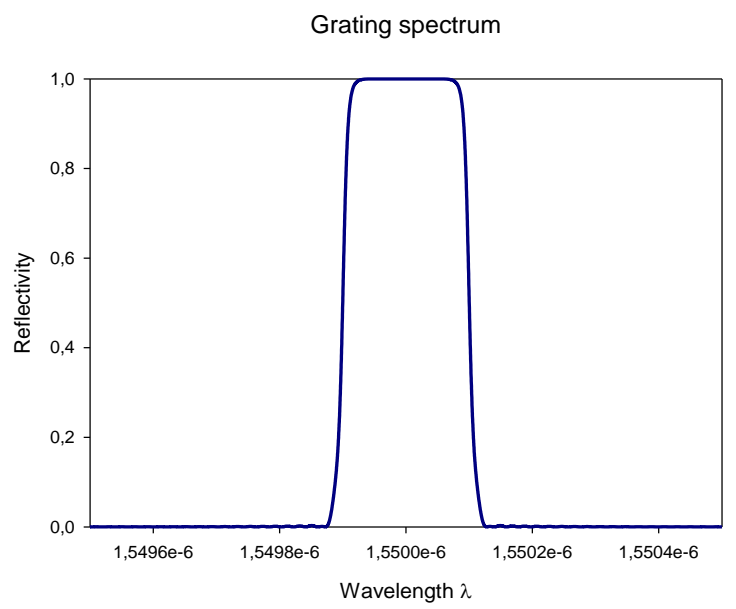

Fig. $8.100 \%$ reflected power of the FBGS using the proposed GUI for the 2-terms profile

\section{Conclusions}

This paper introduced a Graphical User Interface for a novel theoretical approach to optimize the window profile of FBGS using an automated MATLAB-based program. The main objective of this optimization is to calculate automatically the proposed optimized window profile based on the design setting of the FBGS, with the aim to get the higher reflectivity level as long as side lobes are kept below the required level. The proposed easy-to-use graphical interface allows also researchers to determine easily all parameters required for high FBGS performance providing a high precision and accuracy of numerical results. The proposed theoretical approach has been compared with the window profiles proposed by other authors to demonstrate the efficiency of the proposed method. Simulation results shows that the proposed method offers better reflected power for all values of design parameters, which include Grating Length $L$ and Refractive index modulation amplitude $d n$. Finally, a serial calculation and optimization of the proposed 
ÓPTICA PURA Y APLICADA

$\underline{\text { www.sedoptica.es }}$

method resulted in Reflected power of $100 \%$ with SLL and FWHM below $-40 \mathrm{~dB}$ and $0.2 \mathrm{~nm}$, respectively, which has not previously been achieved using any of the state-of-art window profile alternatives. All results show very satisfactory performance of the proposed method. 